\title{
Bibliotheken müssen geöffnet bleiben!
}

\section{Der Deutsche Bibliotheksverband appelliert an Länder und Kommunen}

https://doi.org/10.1515/bd-2021-0017

Im Beschluss der Bund-Länder-Konferenz vom 28.10.2020 werden Bibliotheken als Einrichtungen nicht genannt. Die konkrete Ausgestaltung der Beschlüsse obliegt jetzt den einzelnen Bundesländern. Nach Presseberichten hat Bayern gestern als erstes Bundesland bestätigt, dass Bibliotheken und Büchereien offenbleiben. Auch andere Bundesländer wie Berlin und Sachsen-Anhalt haben dies so in ihren Verordnungen umgesetzt.

Der Deutsche Bibliotheksverband e.V. (dbv) begrüßt diese notwendige Bestätigung außerordentlich. Bibliotheken sind Bildungseinrichtungen, Orte der Wissenschaft und der Kultur. Sie halten ein umfangreiches Medienangebot zur Information für Schule, Studium, Arbeit und die persönliche (Weiter-)Bildung vor. Bibliotheksgesetze in einigen Bundesländern definieren sie als Bildungseinrichtungen und Partner für lebensbegleitendes Lernen. Sie fördern Wissen und gesellschaftliche Integration. Sie stärken die Lese-, Informations- und Medienkompetenz ihrer Nutzer*innen durch geeignete Maßnahmen sowie durch die Zusammenarbeit mit Kindertagesstätten, Schulen und anderen Bildungseinrichtungen.

Bibliotheken gehörten zu den ersten Einrichtungen, die nach dem Lockdown im Frühjahr dieses Jahres ihre Häuser wieder öffnen durften. Sie haben dafür strenge und der jeweiligen Situation angepasste Abstands- und Hygienemaßnahmen entwickelt und Schritt für Schritt insbesondere ihre digitalen Angebote erweitert.

Dazu Andreas Degkwitz, Bundesvorsitzender des dbv: „Gerade jetzt, wo viele harte Einschränkungen für alle notwendig sind, um die zweite Corona-Welle zu brechen, sind Dienstleistungen und Angebote der Bibliotheken ein wichtiger Beitrag zur Daseinsvorsorge. Gerade Familien oder sozial Benachteiligte sind jetzt mehr denn je auf diese Angebote angewiesen, die sie unter strengen Abstandsund Hygienevorkehrungen in Bibliotheken nutzen können. Der Deutsche Bibliotheksverband appelliert an Länder und Kommunen, die Öffentlichen und wissenschaftlichen Bibliotheken geöffnet zu lassen.“ 


\section{Der Deutsche Bibliotheksverband}

Der Deutsche Bibliotheksverband e.V. (dbv) vertritt mit seinen mehr als 2.100 Mitgliedern bundesweit rund 9.000 Bibliotheken mit 25.000 Beschäftigten und 11 Mio. Nutzer^innen. Sein zentrales Anliegen ist es, Bibliotheken zu stärken, damit sie allen Bürger*innen freien Zugang zu Informationen ermöglichen. Der Verband setzt sich ein für die Entwicklung innovativer Bibliotheksleistungen für Wissenschaft und Gesellschaft. Als politische Interessensvertretung unterstützt der dbv die Bibliotheken insbesondere auf den Feldern Informationskompetenz und Medienbildung, Leseförderung und bei der Ermöglichung kultureller und gesellschaftlicher Teilhabe für alle Bürgerinnen.

Mehr Informationen finden Sie unter www.bibliotheksverband.de.

\section{Pressekontakt:}

Kristin Bäßler

Leitung Kommunikation / Pressesprecherin

Deutscher Bibliotheksverband e.V.

Fritschestr. 27-28 | 10585 Berlin

Tel.: 030/644 98 99-25 | E-Mail: baessler@bibliotheksverband.de

URL: www.bibliotheksverband.de 